\title{
A Review on Energy Efficient of Clustering-based Routing Protocol in Wireless Sensor Network
}

\author{
Lalit Kumar Chourse \\ Truba Institute of Engineering \& \\ Information Technology, \\ Bhopal, India
}

\author{
Deepa Chaurse \\ Maulana Azad National \\ Institute of Technology, \\ Bhopal, India
}

\author{
Priyanka Chourse \\ VNS Institute of Technology, \\ Bhopal, India
}

\begin{abstract}
Wireless Sensor Network (WSN) is infrastructure less. Sensors usually rely on their battery for power, which cannot be recharge or replaced. So in this paper, comparisons of energy performance on different position of base station are analyzed and also analyzed LEACH and it's up gradation. Cluster-based routing mechanism use message success rate to guarantee reliability for network. In this mechanism cluster head selection algorithm based on node connectivity and splitting and merging of cluster are performed. A clustering routing algorithm based on gateway in wireless sensor network. The gateway nodes elected ensure the network connectivity using multi-hop for communication between $\mathrm{CHs}$ and sink.
\end{abstract}

\section{Keywords}

Routing, clustering, Energy efficiency, Energy level, Cluster head $\mathrm{CH}$, network lifetime.

\section{INTRODUCTION}

Wireless Sensor Networks (WSNs) are communication networks that interconnect several sensor devices to perform some task or set of tasks. A sensor node is made up of four basic components: a sensing unit, a processing unit, a transceiver unit and a power unit. These sensor nodes can self organize to form a network and can communicate with each other in a wireless manner. Each self-organized node collects data from the environment, exchanges these data with other nodes and sends the final information to the sink node or the base station. Commonly sensors are severely energy constrained since they are battery Powered.

The WSN can be applied to various types of applications, such as environment management and military monitoring. So, it is essential to improve the energy efficiency to enhance the quality of application service [4].

\section{ISSUES IN WSN}

This study focuses on common parameters of well-known cluster based routing protocols. LEACH protocol as a representative to investigate their energy performance sensitivity to the number of clusters, the location of the base station and the data message size.

An Inter-Cluster Route Algorithm is based on the Choice of Gateway (IRCG). The algorithm considers that the nodes are in the case of transmitting power being not adjustable, and its routing is according to using a single hop in intra-cluster communication and inter-cluster communication through reverse aggregation tree constructed by cluster heads and the gateway nodes elected, communicate with the sink node by the way of multi-hop.

\section{LOW-ENERGY ADAPTIVE} CLUSTERING HIERARCHY (LEACH)

Perhaps the first network protocol that is specifically designed for wireless sensors is the Low-Energy Adaptive Clustering Hierarchy (LEACH) protocol [9]. It is also the base protocol from which several of the well-known routing protocols for wireless sensor networks are derived. The main setting that LEACH protocol addresses is that of a large number of homogeneous, resource-constrained cheap, stationary nodes monitoring the environment. Nodes periodically send their readings to a base station located far away from the field as shown in Figure 1.

The protocol achieves its power saving goals by allowing a small percentage of the nodes (cluster heads) to collect data from their surrounding neighbors, aggregate the data and send a single report to the base station representing the combined readings. The protocol avoids depleting the cluster heads energies by selecting a new set of cluster heads at the beginning of each round. The set up overhead is assumed to be negligible since the setup time is small compared to the rounds duration. The protocol uses a randomized routine for each node to elect itself as a cluster head. This routine is run locally at each node to avoid the traffic overhead of a centralized routine

\subsection{Clusters Formation in LEACH:}

LEACH [3] attempts to minimize this communication power dissipation by organizing the network into clusters. In a large WSN, there is a huge amount of data generated that the operator will receive and needs to process. Clustering thus achieves two goals, first, it reduces that amount of data sent to the base station, and second it reduces the power expenditure by the network.

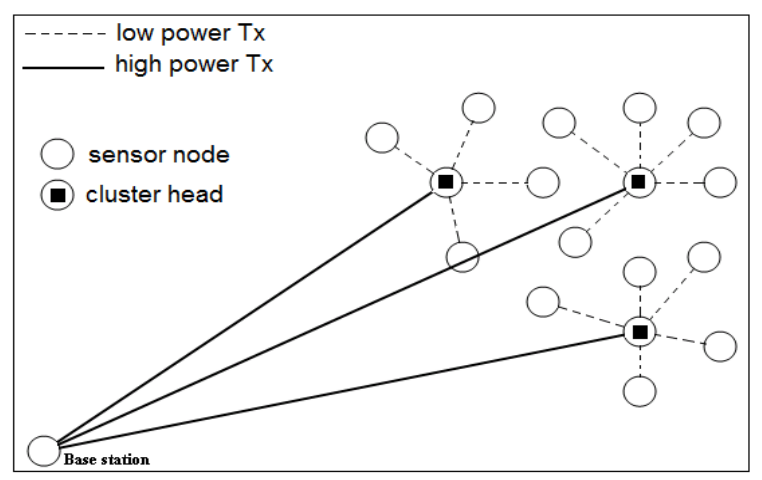

Fig1. Cluster-based Network Routing

LEACH protocol operates in rounds, where in each round a new set of cluster heads is selected. Each round is divided into 
a setup phase and a steady state operation phase. Since this is a distributed system, each node elects itself as a cluster head independently during the setup phase. To do this, each node (n) will independently choose a random number in the interval $(0,1)$. This number is then compared to the network wide threshold $\mathrm{T}(\mathrm{n})$ given by the equation:

$$
T(n)= \begin{cases}\frac{P}{1-P\left(r \bmod \frac{1}{P}\right)} & \text { if } n \in G \\ 0 & \text { otherwise }\end{cases}
$$

Where $\mathrm{p}$ is the optimized cluster heads percentage. This number is determined prior to network deployment. It is calculated using empirical data to get the percentage of cluster heads that gives the lowest energy consumption in the network. $\mathrm{G}$ is the group of nodes that have not been cluster heads in the past 1-p rounds, and $r$ is the round number. In round 0 , all nodes are eligible to elect themselves as cluster heads. During subsequent rounds, the number of eligible nodes decreases, consequently, the threshold probability must increase to maintain the required percentage.

\subsection{Energy Performance of clustering based routing protocol}

According to Ahmed Badi, Imad Mahgoub, Ahmed Badi, Ima Mahgoub [9], they compare the performance of cluster-based protocols. LEACH is the first and the base for most of cluster-based wireless sensor routing protocols. For LEACH, $5 \%$ of the nodes in the network are selected as cluster heads in this performance analysis. The reference scenario for performance comparison is direct transmission in which each node sends its own data directly to the Base Station.

Radio Signal Propagation Model, it can be categorizes as follows:
A. LEACH Energy Performance vs. Direct Transmission
B. Effect of the Number of Clusters on LEACH Energy Performance.
C. Effect of Base Station Location on Energy performance.

\section{Radio Signal Propagation Model:}

Radio transmission power is a function of some positive power of the distance covered. This can be expressed mathematically as follows:

$$
\mathrm{ETx}=\mathrm{f}(\mathrm{dn})
$$

For this analysis, use LEACH first order radio model assumptions, where the transmission and the receiving power are given by the following equations:

$\operatorname{ETx}(\mathrm{k} ; \mathrm{d})=$ Eelect $* \mathrm{k}+\_$Eamp $* \mathrm{~K} * \mathrm{~d} 2$

The receiving energy is considered constant for a given message size and is expressed by the following formula:
Where ' $k$ ' is the number of data bits, ' $d$ ' is the distance between the transmitter and the receiver, ' $n$ ' is the signal attenuation distance exponent factor, 'Eelect' is the power needed to drive the electronic circuitry, and '€amp' is the power needed by the transmission amplifier circuit.

\section{A. LEACH Energy Performance vs. Direct:}

The comparison of LEACH and Direct transmissions, LEACH is more power saving than direct transmission in large networks as shown in figure2.

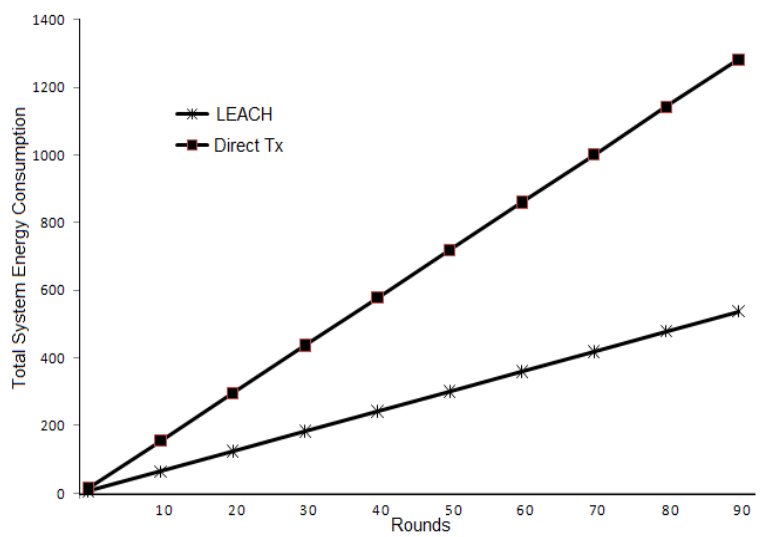

Fig2. LEACH Energy Performance vs Direct Tx.

B. Effect of the Number of Clusters on LEACH Energy Performance:

Compare the energy performance of LEACH for different cluster head percentages for optimal number of clusters that gives the best energy performance.

- The best performance is obtained when the percentage is around $2 \% \sim 3 \%$.

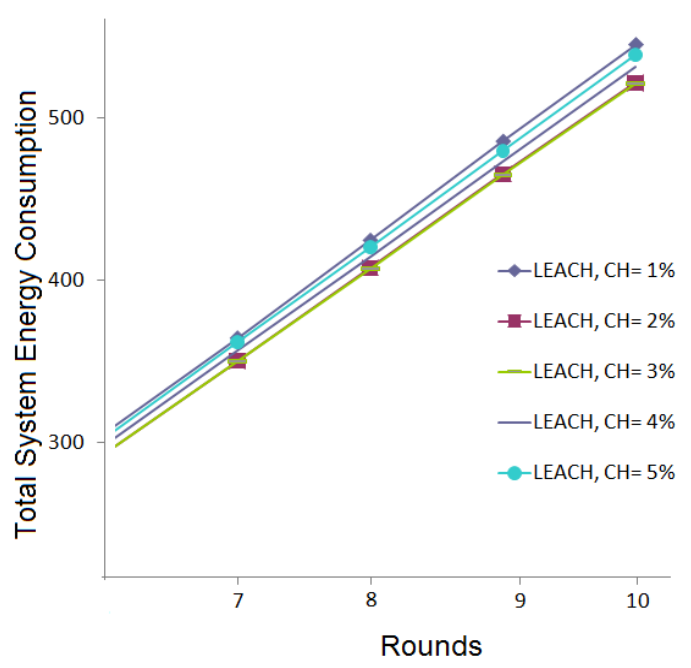

Fig3. Effect of LEACH cluster head $(\mathrm{CH})$ percentage $(1 \%-5 \%)$

$\operatorname{ERx}(\mathrm{k})=$ Eelect $* \mathrm{k}$ 
- In another case the optimal value of $\mathrm{CH}$ percentage is between $5 \%$ to $10 . \%$ [1][3]

Table1: Average Overhead Energy of the network, and the Network lifetime, for different percentages of $\mathrm{CHs}$ in network

\begin{tabular}{|l|l|l|}
\hline \%CH Formation & $\begin{array}{l}\text { \%Overhead } \\
\text { Energy consumed }\end{array}$ & $\begin{array}{l}\text { Energy consumed } \\
\text { in 1000 } \\
\text { rounds(Joule) }\end{array}$ \\
\hline 1 & 74 & 182.1914 \\
\hline 5 & 22 & 198.0256 \\
\hline 10 & 21 & 198.4349 \\
\hline 15 & 24 & 209.8809 \\
\hline 20 & 26 & 220.8879 \\
\hline 25 & 30 & 235.4284 \\
\hline 33 & 31 & 243.5268 \\
\hline
\end{tabular}

The average overhead energy consumed increases when the number of $\mathrm{CH}$ formed increases or decreases from an optimum value of $\mathrm{CH}$ percentage. When number of $\mathrm{CHs}$ increases more than an optimum value say $10 \%$ of the total number of node then there are other things which start affecting the energy utilizations like number of broadcasts and transmission of control packets in contention period etc therefore energy consumed in overhead also starts increasing.

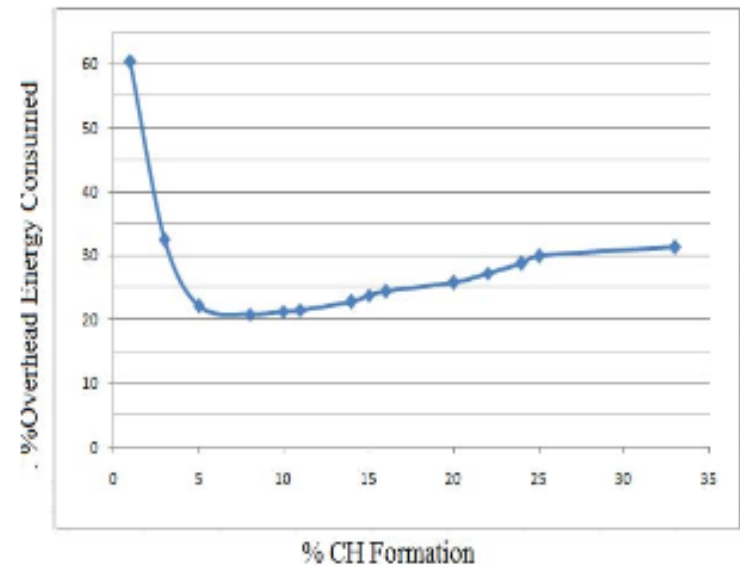

Fig4. Overhead energy Percentage consumed with Probability of CH Formation
C. Effect of Base Station Location on Energy performance.

Table2: Comparisons of energy performance on different positions of base stations

\begin{tabular}{|l|l|l|l|l|}
\hline $\begin{array}{l}\text { Position } \\
\text { of Base } \\
\text { Station }\end{array}$ & $\begin{array}{l}\text { Para } \\
\text { meter }\end{array}$ & Size & Effects & $\begin{array}{l}\text { Leach over } \\
\text { Direct } \\
\text { transmission }\end{array}$ \\
\hline $\begin{array}{l}\text { Outside } \\
\text { the Field }\end{array}$ & - & - & - & Less Power \\
Consumption
\end{tabular}

\subsection{LEACH has following disadvantages [10]}

- In LEACH protocol, the $\mathrm{CH}$ collects and aggregates data from sensors in its own cluster and passes the information to the BS directly. $\mathrm{CH}$ might be located far away from the BS, so it uses most of its energy for transmitting and because it is always on it will die faster than other nodes.

- In LEACH, Each $\mathrm{CH}$ directly communicates with BS no matter the distance between $\mathrm{CH}$ and BS. I will consume lot of its energy if the distance is far.

- LEACH offers no guarantee about the placement and/or number of cluster heads.

- In the original leach, the $\mathrm{CH}$ is always on receiving data from cluster members, aggregate these data and then send it to the BS that might be located far away from it. The $\mathrm{CH}$ will die earlier than the other nodes in the cluster because of its operation of receiving, sending and overhearing. When the $\mathrm{CH}$ die, the cluster will become useless because the data gathered by cluster nodes will never reach the base station. 
Following protocols helps to reduce these disadvantages of LEACH:

\subsubsection{E-LEACH protocol}

- Energy-LEACH protocol improves the $\mathrm{CH}$ selection procedure.

- It makes residual energy of node as the main metric which decides whether the nodes turn into $\mathrm{CH}$ or not after the first round.

- E-LEACH is divided into rounds, in the first round, every node has the same probability to turn into $\mathrm{CH}$, that mean nodes are randomly selected as $\mathrm{CHs}$, in the next rounds, the residual energy of each node is different after one round communication and taken into account for the selection of the $\mathrm{CHs}$.

- That mean nodes have more energy will become a $\mathrm{CHs}$ rather than nodes with less energy.

\subsubsection{TL-LEACH}

- A new version of LEACH called Two-level Leach was proposed. In this protocol; $\mathrm{CH}$ collects data from other cluster members as original LEACH, but rather than transfer data to the BS directly, it uses one of the $\mathrm{CHs}$ that lies between the $\mathrm{CH}$ and the BS as a relay station

\subsubsection{M-LEACH protocol}

- Multi hop-LEACH protocol selects optimal path between the $\mathrm{CH}$ and the $\mathrm{BS}$ through other $\mathrm{CHs}$ and use these $\mathrm{CHs}$ as a relay station to transmit data over through them [2].

- First, multi-hop communication is adopted among CHs. Then, according to the selected optimal path, these $\mathrm{CHs}$ transmit data to the corresponding $\mathrm{CH}$ which is nearest to $\mathrm{BS}$.

- Finally, this $\mathrm{CH}$ sends data to BS. M-LEACH protocol is almost the same as LEACH protocol, only makes communication mode from single hop to multi-hop between $\mathrm{CHs}$ and $\mathrm{BS}$.

\subsubsection{LEACH-C}

- The protocol, called LEACH-C, uses a centralized clustering algorithm and the same steady-state phase as LEACH. LEACH-C protocol can produce better performance by dispersing the cluster heads throughout the network. During the set-up phase of LEACH-C, each node sends information about its current location (possibly determined using GPS) and residual energy level to the sink.

- In addition to determining good clusters, the sink needs to ensure that the energy load is evenly distributed among all the nodes.

- To do this, sink computes the average node energy, and determines which nodes have energy below this average.

- Once the cluster heads and associated clusters are found, the sink broadcasts a message that obtains the cluster head ID for each node.

- If a cluster head ID matches its own ID, the node is a cluster head; otherwise the node determines its TDMA slot for data transmission and goes sleep until its time to transmit data.
- The steady-state phase of LEACH-C is identical to that of the LEACH protocol.

\subsubsection{V-LEACH}

- In our new version of LEACH protocol, the cluster contains; $\mathrm{CH}$ (responsible only for sending data that is received from the cluster members to the $\mathrm{BS}$ ), vice- $\mathrm{CH}$ (the node that will become a $\mathrm{CH}$ of the cluster in case of $\mathrm{CH}$

- In the original leach, the $\mathrm{CH}$ is always on receiving data from cluster members, aggregate these data and then send it to the BS that might be located far away from it. The $\mathrm{CH}$ will die earlier than the other nodes in the cluster because of its operation of receiving, sending and overhearing.

- When the $\mathrm{CH}$ die, the cluster will become useless because the data gathered by cluster nodes will never reach the base station.

- In V-LEACH protocol, besides having a $\mathrm{CH}$ in the cluster, there is a vice- $\mathrm{CH}$ that takes the role of the $\mathrm{CH}$ when the $\mathrm{CH}$ dies.

\subsubsection{EFFICIENT-ROUTING LEACH (ER- LEACH)}

Efficient Routing LEACH (ER-LEACH) [2] which is a modified version of LEACH protocol ; there are three contributions in this protocol which are: Enhancing the selection of the cluster head during setup phase by taking into account the residual energy of any sensor node which intends to become a $\mathrm{CH}$ to prolong the network lifetime.

The second contribution is trying to reduce the overhead of dynamic clusters generation by using alternative $\mathrm{CH}$ which is expected to take the role of the $\mathrm{CH}$ in case that the underlying $\mathrm{CH}$ died which will prolong the lifetime of each cluster.

Finally for the sake of load balancing the zone routing protocol is used which attempts to balance the load over $\mathrm{CHs}$ evenly by permitting the $\mathrm{CH}$ to discover the optimal route to the BS with less cost messages update and then sends the fused data to the BS through many other $\mathrm{CHs}$ instead of direct sending to the BS. ER-LEACH is expected to perform well especially when the mobility is very high and will prolong the overall network lifetime through load balancing.

\subsubsection{LEACH - SPARE MANAGEMENT (LEACH-SM)}

LEACH-SM protocol [2] modifies LEACH by enhancing it with an efficient management of spares. It is also designed for static sensor nodes and static targets. LEACH-SM deals with both energy-consumption inefficiencies of LEACH. Identification of spares alone increases the overall WSN lifetime. LEACH-SM adds a phase, called the spare selection phase, to the original LEACH protocol. It follows the setup phase, and is followed by the regular operation of the WSN. (Regular WSN operation is divided into frames, during which nodes follow cycles of awake and nap intervals.)

The Decentralized Energy-efficient Spare Selection Technique (DESST) is run during this spare selection phase. DESST, run in parallel on all WSN nodes in all clusters, allows each node to decide whether it should become a spare. It is done in such a way that the above-threshold target coverage is maintained by the WSN. All spares go asleep to conserve energy. As the result, WSN's lifetime is extended. 
Table 3 indicates the comparison of various LEACHes

\begin{tabular}{|c|c|c|c|c|}
\hline Protocols & CH Selection & Data Transmission & Mobility & $\begin{array}{l}\text { Location } \\
\text { Awareness }\end{array}$ \\
\hline LEACH & Random & All CH sends directly to BS & Stationary & No \\
\hline LEACH-C & $\begin{array}{l}\text { CH Selection done by BS based on } \\
\text { energy }\end{array}$ & The chosen $\mathrm{CH}$ sends directly to BS & Stationary & Yes \\
\hline TL-LEACH & Periodically & $\begin{array}{l}\text { Rather than transfer data to the BS directly, } \\
\text { it uses one of the } \mathrm{CHs} \text { that lies between the } \\
\mathrm{CH} \text { and the BS as a relay station. }\end{array}$ & Stationary & No \\
\hline E-LEACH & Random & $\begin{array}{l}\text { Nodes have more energy will become a CHs } \\
\text { rather than nodes with less energy. }\end{array}$ & Stationary & No \\
\hline V-LEACH & Random & $\begin{array}{l}\text { If the base station is not within range of } \mathrm{CH} \text {, } \\
\text { it is routed through other } \mathrm{Hs} \text {. There is also } \\
\text { an alternative } \mathrm{CH} \text { in case of death. }\end{array}$ & Mobile & Yes \\
\hline M-LEACH & Random & $\begin{array}{c}\mathrm{CHs} \text { transmit data to the corresponding } \mathrm{CH} \\
\text { which is nearest to BS }\end{array}$ & Stationary & No \\
\hline $\begin{array}{c}\text { ER-LEACH } \\
.\end{array}$ & $\begin{array}{l}\text { CH Selection based on Energy } \\
\text { decided within the cluster }\end{array}$ & $\begin{array}{l}\text { If the base station is not within range of } \mathrm{CH} \text {, } \\
\text { it is routed through other } \mathrm{CH} \text {. There is also } \\
\text { an alternative } \mathrm{CH} \text { in case of death }\end{array}$ & Mobile & Yes \\
\hline LEACH-SM & $\begin{array}{c}\text { During the cluster setup phase, } \\
\text { each node decides in parallel } \\
\text { whether it should become an active } \\
\text { primary node or a passive spare } \\
\text { node. }\end{array}$ & $\begin{array}{c}\text { The passive spare nodes go asleep where the } \\
\text { data is transmitted through the primary } \\
\text { active node. }\end{array}$ & $\begin{array}{l}\text { Designed for } \\
\text { static sensor } \\
\text { nodes and } \\
\text { static targets }\end{array}$ & Yes \\
\hline
\end{tabular}




\section{CLUSTER HEAD SELECTION}

Overhead and non consideration of communication ranges a cluster head are basic problem in LEACH and its type. For making good routing algorithm following steps has to be considered network information generation, cluster head selection, cluster formation, and cluster management [2].

\subsection{Network information generation}

By using Message Success Rate (MSR), nodes generating network information and sends information to adjacent nodes. Routing should take place by using multi-hop to sink node.

Definition 1: MSR can be determined as given below. Here let sPacekt be sending packet to neighbor, rPacket be number of receiving packet in neighbor, and $\mathrm{t}$ be the total time of communicate between neighboring nodes.

\section{AvgSuccRate $=$ rPacket $(\mathrm{t}) /$ sPacket $(\mathrm{t})$}

Using MSR, sensor nodes set the parent-child node and the routing path to the sink node through the parent nodes. This resembles MST (Maximum Spanning Tree) technique. And each sensor node produces its own network information through the routing path. The network information of a sensor node is generated by communicating it with adjacent nodes.

\subsection{Cluster head Selection}

This phase selects cluster header node by considering the routing path and network information. All nodes in a cluster transmit data through the cluster head. The optimal rate of cluster head means the minimum number of head nodes in a cluster for reducing amount of energy consumption efficiently.

For cluster head selection, the first step is to provide high connectivity between a cluster head and neighboring nodes that means density of the cluster head node has to be high.

Secondly, the message success rate has to be guaranteed to be higher than a given threshold.

Finally, a cluster head has to be positioned in the center of the network so that the number of hops may be uniform.

Definition 2: Weight of cluster head can be determined as below.

Weight $=\alpha^{*}$ NeighborCount $+\beta * \Sigma($ NeighborMSR $)$

$$
\text { - } \gamma * \text { HopCount }
$$

Where,

NeighborCount $=$ number of neighbors.

MsgSuccRate $=$ message success rate between neighboring nodes.

HopCount $=$ number of hops .

$\alpha, \beta$ and $\gamma=$ threshold value for the cluster head selection

$$
(\alpha+\beta+\gamma=1)
$$

\subsection{Cluster formation}

For Cluster formation steps are: initial clusters, splitting and merging algorithms to generate the optimal cluster

First, the initial cluster calculate optimal rate of cluster head:

Optimal rate $=$ no. of nodes $/$ rate of cluster head

Parent node gets information from leaf node about node count and check whether the count is greater than optimal cluster rate. When node are satisfying optimal cluster rate then Cluster is constructed.

Splitting has to be done because some initial cluster do not satisfy splitting rate. On the basis of child node's information, parent node chooses those part of cluster member which have more lower nodes than other one and put it into new cluster .Therefore clusters have even number of nodes.

Finally, the cluster merging step: merge clusters which do not satisfy the merging threshold. To merge adjacent clusters, it considers the number of an adjacent cluster's members.

After cluster formation, inter cluster routing mechanism (multi-hop routing) is used. In multi hop routing, heads can transfer data to each other by using gateway when they are in communication range [11].

\section{ELECTION OF GATEWAY NODE 5.1 The method for selecting gateway nodes is as follows [8]:}

(1) In clustering phase, each cluster head broadcasts Sensor_Msg, while the other nodes keep receiving state and receive the message sent by $\mathrm{CHs}$.

(2) If a node received more than one Sensor_Msg from CHs, it will change its state to 'Red', marked itself as the gateway node.

\subsection{Construction of Routing Gathering Tree}

The main idea of IRCG about the construction of routing tree: The cluster member communicate with $\mathrm{CHs}$ using a single hop, and the inter-cluster communication through the reverse gathering tree[8] which is built by the $\mathrm{CHs}$ and gateway as key point, sink as the root.

The gathering tree constructed by the way of "cluster head $\rightarrow$ Gateway $\rightarrow$ cluster head node" repeatedly until reach the sink. During the cluster communication, the other nodes keep sleep to save energy. After each node have been clustered, then the clock for building gathering tree is triggered, and the construction algorithm based on reverse diffusion is started.

The detail of the construction of gathering tree is as follows: First, initializing, set the hop number of sink to 0 and broadcast message containing the hops number (from Itself to sink node).The adjacent node receives the message, then make sink as its parent node, while hops number add 1, and then continue to broadcast message.

Nodes which received the message, determine its state whether different from the state of the node which sending messages (ego it is cluster head, the node which sending packets is also cluster head). If the states is the same, then abort the message; else, make the sender node as its parent, 
while hops number add 1, and then continue to broadcast message. So it can ensure the gathering tree is built by cluster head and Gateway alternatively.

Node receives more than one message at the same time, select the sender had strongest signal as the parent. This process has been spread throughout the whole network, so that all nodes are aware of their parent and the number of hops to the sink. In this way, the routing tree can be constructed well

In each round of the execution of IRCG algorithm, after the initialization phase, cluster members send the information to $\mathrm{CHs}$ according to the TDMA time slot. $\mathrm{CHs}$ aggregate the data and then send to sink through the routing tree.

\section{CONCLUSION}

In this paper, the effect of base station location relative to the field and the effect of message size are addressed and a cluster-based routing mechanism use message success rate to guarantee reliability for network. In this mechanism cluster head selection algorithm based on node connectivity and splitting and merging of cluster are performed. To reduce data communication overhead, information of neighbor nodes at both cluster construction and cluster head selection are used. A clustering routing algorithm based on gateway in wireless sensor network. The gateway nodes elected ensure the network connectivity using multi-hop for communication between $\mathrm{CHs}$ and sink. In future we use optimal parameters to reduce re-cluster formation by putting weights and suggesting different cluster head selection.

\section{REFERENCES}

[1] Rabia Noor Enam , Mumtazul Imam and Rehan Inam Qureshi, "Energy Consumption in Random Cluster Head selection Phase of WSN", 2012 IACSIT Hong Kong Conferences, IPCSIT vol. 30 (2012), IACSIT Press, Singapore.

[2] Sabarish B A, Guru Moorthy S M, Dhivya M A, Sakthi Naveen K, Vaishnavi S "A survey on clustering protocols in Wireless Sensor Networks" International Journal Of Advances In Computing and Information Technology, published on April 2012.

[3] Anindita Ray, Debashis De, "P-EECHS: Parametric Energy Efficient Cluster Head Selection protocol for
Wireless Sensor Network", International Journal of Advanced Computer Engineering \& Architecture, Vol. 2 No. 2, June-December, 2012

[4] Moufida Maimour, Houda Zeghilet, "A Survey Paper on Cluster-based Routing Protocols for Energy Efficiency in Wireless Sensor Networks" Francis Lepage CRAN laboratory, Nancy University, CNRS France

[5] Min Yoon, Jaewoo Chang, "Design and Implementation of Cluster-based Routing Protocol using Message Success Rate in Sensor Networks," 2011 IEEE

[6] International Conference on High Performance Computing and Communications.

[7] Ahmed Badi, Imad Mahgoub, Ahmed Badi, Ima Mahgoub, "Impact of Transmission-related Parameters on the Energy Performance of Cluster-based Routing Protocols for Wireless Sensor Networks," 2011 International Conference on Innovations in Information Technology.

[8] Li Peng", Jian-bo xc', Wei Liang, "Inter-Cluster Route Algorithm Based on the Gateway for Wireless Sensor Networks," 2011 International Conference on Electronic \&Mechanical Engineering and Information Technology.

[9] M. Bani Yassein, A. Al-zou'bi, Y. Khamayseh, W. Mardini "Improvement on LEACH Protocol of Wireless Sensor Network (VLEACH)", International Journal of Digital Content Technology and its Applications Volume 3, Number 2, June 2009.

[10] F. Xiangning, S. Yulin, "Improvement on LEACH Protocol of Wireless Sensor Network", International Conference on Sensor Technologies and Applications, IEEE, pp.260-264, 2007

[11] Younis M, Youssef M, Arisha K. "Energy -Aware routing in cluster-based sensor networks". In: Proc. of the 10th IEEE Int'1 Symp. on Modeling, Analysis and Simulation of Computer and Telecommunications Systems. Fort Worth: IEEE Computer Society, 2002.129136. 